\title{
Optimal Shape Control of Piezoelectric Intelligent Structure Based on Genetic Algorithm
}

\author{
Zhanxi Wang, ${ }^{1,2}$ Xiansheng Qin, ${ }^{1}$ Shunqi Zhang, ${ }^{1}$ Jing Bai, ${ }^{1}$ Jing Li, ${ }^{1}$ and Genjie $\mathbf{Y u}^{2}$ \\ ${ }^{1}$ School of Mechanical Engineering, Northwestern Polytechnical University, Xian 710072, China \\ ${ }^{2}$ Xinlian College, Henan Normal University, Zhengzhou 451400, China \\ Correspondence should be addressed to Zhanxi Wang; zxwang@nwpu.edu.cn
}

Received 29 September 2016; Revised 12 April 2017; Accepted 27 August 2017; Published 11 October 2017

Academic Editor: Cristina Leonelli

Copyright (c) 2017 Zhanxi Wang et al. This is an open access article distributed under the Creative Commons Attribution License, which permits unrestricted use, distribution, and reproduction in any medium, provided the original work is properly cited.

\begin{abstract}
Shape variation induced by mismachining tolerance, humidity and temperature of the working environment, material wear and aging, and unknown external load disturbances have a relatively large influence on the dynamic shape of a mechanical structure. When integrating piezoelectric elements into the main mechanical structure, active control of the structural shape is realized by utilizing the inverse piezoelectric effect. This paper presents a mathematical model regarding piezoelectric intelligent structure shape control. We also applied a genetic algorithm, and given a piezoelectric intelligent cantilever plate with both ends affected by a certain load, optimal shape control results of piezoelectric materials were analyzed from different perspectives (precision reference or cost reference). The mathematical model and results indicate that, by optimizing a certain number of piezoelectric actuators, high-precision active shape control can be realized.
\end{abstract}

\section{Introduction}

A variety of high-speed and high-precision mechanical structures require extremely precise shape and position when in operation, and slight variations greatly influence the dynamic performances of these structures $[1,2]$. For instance, mismachining tolerance, humidity and temperature of the working environment, material wear and aging, and unknown external load disturbances may affect the shape of a structure. Consequently, active shape control and compensation are required in real operation. Piezoelectric elements are integrated into the main structure, the inverse piezoelectric effect is utilized, and piezoelectric elements are deformed via an external voltage, which is passed on to the main structure, thus realizing active control of structural shape.

Due to structure complexity and the inherent electromechanical coupling effect of a piezoelectric intelligent structure, studies on structural shape control are typically based on the mathematical mode of the intelligent structure using a finite element method. Following conclusions toward the theory of intelligent structure, Wada et al. [3] proposed a shape-variable and self-adaptive intelligent structure framework to meet future requirements of structures like space stations, aircrafts, and satellites. Based on a hierarchical theory, Donthireddy and Chandrashekhara [4] established a finite element numerical model of laminated piezoelectric beams, studied the shape control problem of these beam, and analyzed the influence of stacking sequence and boundary conditions on shape control. Varadarajan et al. [5] performed shape control of laminated composite plates with piezoelectric actuators, and using the minimum error function of ideal shape and control shape, voltage distribution was optimized and an active feedback control algorithm was established. Conversely, Chee et al. [6,7] established the static shape control equation of intelligent composite piezoelectric beams and plates based on a high-order displacement function, and classic beam and plate theory, respectively. Furthermore, they applied the generalized function to analyze the specific control effect. Lastly, Lin and Nien [8] established the finite element model of laminated plate shape control using piezoelectric actuators, considered the influence of actuator and sensor locations on shape control, calculated structural stress, and concluded that internal stress of the piezoelectric actuator has huge influence on shape control.

It is important to optimize the design of embedded depth, thickness, and location of piezoelectric elements, as well as 
control voltage, for realizing the shape control effect. Based on a nonlinear constitutive piezoelectric equation and according to the error function and control energy, Sun and Tong [9] utilized the finite element method and Lagrange multiplier method to define an optimization algorithm for piezoelectric actuators on the control voltage of static nonlinear deformation control, and further demonstrated the effectiveness of this algorithm through simulation experiments. On the other hand, Barboni et al. [10] used a method combining a dynamic influence function and closed-loop feedback to study the optimal location of a pair of piezoelectric actuators, thus maximizing piezoelectric intelligent beam displacement. Another study considered the influence of the location, dimension, and voltage of piezoelectric actuators on shape control, and performed multi-objective optimization of cantilever shape control, minimizing beam deflection under the external load effect [11]. Conversely, Zhang et al. [12] studied the integrated optimization configuration problem of piezoelectric actuators and sensors in a flexible structure system by applying the genetic algorithm, while Da Mota Silva et al. [13] used the variance between the preset and real displacement of a specific node to study the static shape control of self-adaptable structure, established an infinite model of the structure system, and determined the optimal driving voltage of piezoelectric elements by applying the genetic algorithm. Experiments verified the applicability and effectiveness of this algorithm. In contrast, based on the shear deformation beam theory and linear piezoelectric theory, Hadjigeorgiou et al. [14] established a numerical model of piezoelectric beams, and utilized the genetic optimization algorithm to optimize the voltage of piezoelectric actuator elements. Numerical results indicated that fewer piezoelectric driving elements achieved the same expected deformation effect following optimization.

In this paper, the piezoelectric intelligent structure was applied to perform active shape control, and the effect location, geometric parameters, and working voltage were solved based on the expected shape or displacement value. Thus, this is a typical multiobjective optimization problem. First, according to the shape function of the piezoelectric intelligent structure and the finite dynamic function, the relationship between the shape or displacement of the structure and its piezoelectric properties, function location, geometric parameters, or input voltage under the influence of constant mechanical load was deduced. Then, from different perspectives (precision preference or cost preference), proper object function was selected to perform multiobjective optimization of the number, function location, and working voltage of the piezoelectric driving elements, thus providing optimal shape control results that satisfy precision requirements.

\section{Piezoelectric Intelligent Structure Shape Control Theory}

2.1. Shape Function and Finite Element Equation. The top and bottom layers of the piezoelectric intelligent plate are symmetrically bonded with piezoelectric actuators, which are applied to produce control bending moments and detect shape change. Because the thickness of these piezoelectric actuators is assumed to be very small as compared with the thickness of the plates, the structure changes due to these piezoelectric layers can be considered negligible. The piezoelectric intelligent plate is modeled by using the fourcorner-node rectangular plate elements, which include four bending degrees of freedom $\left(w, w_{x}, w_{y}, w_{x y}\right)$ at each corner node. According to the Hermitian interpolation polynomial relationship between dimensionless natural coordinates $\xi$ and $\eta$, the relative displacement in any point inside the rectangular element can be expressed as follows:

$$
\begin{gathered}
w(\xi, \eta)=\sum_{i=1}^{4}\left[G_{i}(\xi) G_{i}(\eta) w_{i}+H_{i}(\xi) G_{i}(\eta)\left(\frac{\partial w}{\partial x}\right)_{i}\right. \\
\left.+G_{i}(\xi) H_{i}(\eta)\left(\frac{\partial w}{\partial y}\right)_{i}+G_{i}(\xi) G_{i}(\eta)\left(\frac{\partial w}{\partial z}\right)_{i}\right]
\end{gathered}
$$

where

$$
\begin{aligned}
& G_{i}(\xi)=\frac{1}{4}\left(-\xi_{i} \xi^{3}+3 \xi_{i} \xi+2\right), \\
& H_{i}(\xi)=\frac{1}{4}\left(\xi^{3}+\xi_{i} \xi^{2}-\xi-\xi_{i}\right) .
\end{aligned}
$$

The relationship between natural coordinates and geometric coordinates is

$$
\begin{aligned}
& \xi=2\left(\frac{x}{a}\right)-1, \\
& \eta=2\left(\frac{y}{b}\right)-1,
\end{aligned}
$$

where $a$ and $b$ are the length and width of rectangular element, respectively.

The shape function is expressed according to

$$
\begin{aligned}
& f_{1}(x)=G_{1}(\xi)=G_{4}(\xi), \\
& f_{1}(y)=G_{1}(\eta)=G_{2}(\eta), \\
& f_{2}(x)=G_{2}(\xi)=G_{3}(\xi), \\
& f_{2}(y)=G_{3}(\eta)=G_{4}(\eta), \\
& f_{3}(x)=H_{1}(\xi)=H_{4}(\xi), \\
& f_{3}(y)=H_{1}(\eta)=H_{2}(\eta), \\
& f_{4}(x)=H_{2}(\xi)=H_{3}(\xi), \\
& f_{4}(y)=H_{3}(\eta)=H_{4}(\eta) .
\end{aligned}
$$

After substituting (2), (3), and (4) into (1), the displacement of any point inside the finite element is expressed as follows:

$$
w(x, y)=\sum_{i=1}^{16} f_{i}(x, y) q_{i}
$$


where $q_{i}=\left\{w_{1}, w_{1 x}, w_{1 y}, w_{1 x y}, \ldots, w_{4}, w_{4 x}, w_{4 y}, w_{4 x y}\right\}$, and $f_{i}(x, y)$ represents the shape functions of a mode in every degree of freedom, which are listed below:

$$
\begin{aligned}
& f_{1}(x, y)=f_{1}(x) f_{1}(y) \\
& =\frac{1}{a^{3} b^{3}}\left(a^{3}+2 x^{3}-3 a x^{2}\right)\left(b^{3}+2 y^{3}-3 b y^{2}\right), \\
& f_{2}(x, y)=f_{2}(x) f_{1}(y) \\
& =\frac{1}{a^{3} b^{3}}\left(a x^{3}-2 a^{2} x^{2}+a^{3} x\right)\left(b^{3}+2 y^{3}-3 b y^{2}\right), \\
& f_{3}(x, y)=f_{1}(x) f_{2}(y) \\
& =\frac{1}{a^{3} b^{3}}\left(a^{3}+2 x^{3}-3 a x^{2}\right)\left(b y^{3}-2 b^{2} y^{2}+b^{3} y\right), \\
& f_{4}(x, y)=f_{2}(x) f_{2}(y) \\
& =\frac{1}{a^{3} b^{3}}\left(a x^{3}-2 a^{2} x^{2}+a^{3} x\right)\left(b y^{3}-2 b^{2} y^{2}+b^{3} y\right), \\
& f_{5}(x, y)=f_{3}(x) f_{1}(y) \\
& =\frac{1}{a^{3} b^{3}}\left(3 a x^{2}-2 x^{3}\right)\left(b^{3}+2 y^{3}-3 b y^{2}\right), \\
& f_{6}(x, y)=f_{4}(x) f_{1}(y) \\
& =\frac{1}{a^{3} b^{3}}\left(a x^{3}-a^{2} x^{2}\right)\left(b^{3}+2 y^{3}-3 b y^{2}\right), \\
& f_{7}(x, y)=f_{3}(x) f_{2}(y) \\
& =\frac{1}{a^{3} b^{3}}\left(3 a x^{2}-2 x^{3}\right)\left(b y^{3}-2 b^{2} y^{2}+b^{3} y\right), \\
& f_{8}(x, y)=f_{4}(x) f_{2}(y) \\
& =\frac{1}{a^{3} b^{3}}\left(a x^{3}-a^{2} x^{2}\right)\left(b y^{3}-2 b^{2} y^{2}+b^{3} y\right), \\
& f_{9}(x, y)=f_{3}(x) f_{3}(y) \\
& =\frac{1}{a^{3} b^{3}}\left(3 a x^{2}-2 x^{3}\right)\left(3 b y^{2}-2 y^{3}\right), \\
& f_{10}(x, y)=f_{4}(x) f_{3}(y) \\
& =\frac{1}{a^{3} b^{3}}\left(a x^{3}-a^{2} x^{2}\right)\left(3 b y^{2}-2 y^{3}\right), \\
& f_{11}(x, y)=f_{3}(x) f_{4}(y) \\
& =\frac{1}{a^{3} b^{3}}\left(3 a x^{2}-2 x^{3}\right)\left(b y^{3}-b^{2} y^{2}\right), \\
& f_{12}(x, y)=f_{4}(x) f_{4}(y) \\
& =\frac{1}{a^{3} b^{3}}\left(a x^{3}-a^{2} x^{2}\right)\left(b y^{3}-b^{2} y^{2}\right), \\
& f_{13}(x, y)=f_{1}(x) f_{3}(y) \\
& =\frac{1}{a^{3} b^{3}}\left(a^{3}+2 x^{3}-3 a x^{2}\right)\left(3 b y^{2}-2 y^{3}\right),
\end{aligned}
$$

$$
\begin{aligned}
& f_{14}(x, y)=f_{2}(x) f_{3}(y) \\
& =\frac{1}{a^{3} b^{3}}\left(a x^{3}-2 a^{2} x^{2}+a^{3} x\right)\left(3 b y^{2}-2 y^{3}\right), \\
& f_{15}(x, y)=f_{1}(x) f_{4}(y) \\
& =\frac{1}{a^{3} b^{3}}\left(a^{3}+2 x^{3}-3 a x^{2}\right)\left(b y^{3}-b^{2} y^{2}\right), \\
& f_{16}(x, y)=f_{2}(x) f_{4}(y) \\
& =\frac{1}{a^{3} b^{3}}\left(a x^{3}-2 a^{2} x^{2}+a^{3} x\right)\left(b y^{3}-b^{2} y^{2}\right) .
\end{aligned}
$$

The linear constitutive equations of the piezoelectric material can be written as [15]

$$
\begin{aligned}
& \boldsymbol{\sigma}=\mathbf{c}^{\mathrm{E}} \boldsymbol{\varepsilon}-\mathbf{e}^{T} \mathbf{E}, \\
& \mathbf{D}=e \boldsymbol{\varepsilon}+\epsilon^{\mathrm{S}} \mathbf{E},
\end{aligned}
$$

where $\mathbf{c}^{\mathbf{E}}$ is the elastic stiffness matrix, the superscript $T$ denotes transpose, $\mathbf{E}$ is the electric field vector, $\mathbf{D}$ is the electric displacement vector, $\epsilon^{\mathbf{S}}$ is the dielectric constant matrix, and $e$ is the piezo stress/charge constant.

The finite element dynamic equation of the piezoelectric intelligent structure is expressed as follows:

$$
\begin{aligned}
& {\left[\begin{array}{ll}
\mathbf{M} & 0 \\
\mathbf{0} & \mathbf{0}
\end{array}\right]\left\{\begin{array}{l}
\ddot{\mathbf{q}} \\
\ddot{\boldsymbol{\phi}}
\end{array}\right\}+\left[\begin{array}{ll}
\mathbf{C} & \mathbf{0} \\
\mathbf{0} & \mathbf{0}
\end{array}\right]\left\{\begin{array}{l}
\dot{\mathbf{q}} \\
\dot{\boldsymbol{\phi}}
\end{array}\right\}+\left[\begin{array}{ll}
\mathbf{K}_{q q} & \mathbf{K}_{q \phi} \\
\mathbf{K}_{\phi q} & \mathbf{K}_{\phi \phi}
\end{array}\right]\left\{\begin{array}{l}
\mathbf{q} \\
\boldsymbol{\phi}
\end{array}\right\}} \\
& \quad=\left\{\begin{array}{l}
\mathbf{F} \\
\mathbf{Q}
\end{array}\right\},
\end{aligned}
$$

where $\mathbf{q}$ and $\phi$ are the displacement vector and potential vector of each mode in the overall structure, respectively; $\mathbf{M}$, $\mathbf{C}$, and $\mathbf{K}_{q q}$ are the overall weight, resistance, and stiffness matrix of the integrated piezoelectric intelligent structure, respectively; $\mathbf{K}_{q \phi}=\mathbf{K}_{\phi q}^{T}$ is the overall force-electricity coupled stiffness matrix of the integrated piezoelectric material; $\mathbf{K}_{\phi \phi}$ is the overall dielectric stiffness matrix of the piezoelectric material; and $\mathbf{F}$ and $\mathbf{Q}$ are the structure's external force load vector and electric load vector, respectively. The detailed derivation of these element matrices can be found in [16].

Taking into consideration the active static shape control of the piezoelectric intelligent structure, the dynamic response term in the equation is neglected, and (8) then becomes (9). This can then be rewritten as (10):

$$
\begin{aligned}
{\left[\begin{array}{cc}
\mathbf{K}_{q q} & \mathbf{K}_{q \phi} \\
\mathbf{K}_{\phi q} & \mathbf{K}_{\phi \phi}
\end{array}\right]\left\{\begin{array}{l}
\mathbf{q} \\
\boldsymbol{\phi}
\end{array}\right\} } & =\left\{\begin{array}{l}
\mathbf{F} \\
\mathbf{Q}
\end{array}\right\}, \\
\mathbf{K}_{q q} \mathbf{q}+\mathbf{K}_{q \phi} \boldsymbol{\phi} & =\mathbf{F}, \\
\mathbf{K}_{\phi q} \mathbf{q}+\mathbf{K}_{\phi \phi} \phi & =\mathbf{Q} .
\end{aligned}
$$


2.2. Shape Control Mechanism of the Intelligent Structure. The static condensation method was applied to simplify (10) to obtain

$$
\mathbf{K q}=\mathbf{F}+\mathbf{F}_{\phi} \text {, }
$$

where

$$
\begin{aligned}
\mathbf{K} & =\mathbf{K}_{q q}-\mathbf{K}_{q \phi} \mathbf{K}_{\phi \phi}^{-1} \mathbf{K}_{\phi q}, \\
\mathbf{F}_{\phi} & =-\mathbf{K}_{q \phi} \mathbf{K}_{\phi \phi}^{-1} \mathbf{Q} .
\end{aligned}
$$

From (11), the external load of the piezoelectric intelligent structure system consists of 2 parts: the mechanical load $\mathbf{F}$ and electric load $\mathbf{F}_{\phi}$. The electric load is determined by the dielectric properties, geometric parameters, and input voltage of the piezoelectric driving element. Under the condition that the mechanical load remains constant, because of the inverse piezoelectric effect, the displacement of the structure under control can be changed by changing the driving element input voltage changes.

The external electric field of the piezoelectric intelligent structure's sensing unit is zero, and therefore, the potential output is obtained by the second term in (10):

$$
\boldsymbol{\phi}=-\mathbf{K}_{\phi \phi}^{-1} \mathbf{K}_{\phi q} \mathbf{q}
$$

where $-\mathbf{K}_{\phi \phi}^{-1} \mathbf{K}_{\phi q}$ is defined as the displacement sensitivity matrix.

Expressions for stiffness matrix $\mathbf{K}_{q q}^{e}$, force-electric coupling stiffness matrix $\mathbf{K}_{q \phi}^{e}$, and unit dielectric matrix $\mathbf{K}_{\phi \phi}^{e}$ in the finite unit of the piezoelectric intelligent structure are as follows:

$$
\begin{aligned}
\mathbf{K}_{q q}^{e} & =\int_{V}\left[B_{q}\right]^{T}\left[c^{E}\right]\left[B_{q}\right] d V, \\
\mathbf{K}_{q \phi}^{e} & =\int_{V}\left[B_{q}\right]^{T}[e]^{T}\left[B_{q}\right] d V, \\
\mathbf{K}_{\phi \phi}^{e} & =\int_{V}\left[B_{\phi}\right]^{T}\left[\epsilon^{S}\right]\left[B_{\phi}\right] d V,
\end{aligned}
$$

where matrices $\left[B_{q}\right]$ and $\left[B_{\phi}\right]$ consist of partial derivatives of the displacement shape function $\mathbf{f}_{q}$ and potential shape function $\mathbf{f}_{\phi}$ of the unit along the displacement and potential directions, respectively,

$$
\begin{aligned}
& {\left[B_{q}\right]=\left[\begin{array}{lll}
\frac{\partial^{2} \mathbf{f}_{q}}{\partial x^{2}} & \frac{\partial^{2} \mathbf{f}_{q}}{\partial y^{2}} & \frac{\partial^{2} \mathbf{f}_{q}}{\partial z^{2}}
\end{array}\right]^{T},} \\
& {\left[B_{\phi}\right]=\left[\begin{array}{lll}
\frac{\partial \mathbf{f}_{\phi}}{\partial x} & \frac{\partial \mathbf{f}_{\phi}}{\partial y} & \frac{\partial \mathbf{f}_{\phi}}{\partial z}
\end{array}\right]^{T} .}
\end{aligned}
$$

According to the boundary conditions, (14) are integrated to obtain the overall stiffness matrix $\mathbf{K}_{q q}$, force-electric coupling stiffness matrix $\mathbf{K}_{q \phi}$, and dielectric matrix $\mathbf{K}_{\phi \phi}$. From (14), the force-electric coupling stiffness matrix $\mathbf{K}_{q \phi}$ and dielectric matrix $\mathbf{K}_{\phi \phi}$ of the system are not only influenced by parameters of the material itself, but also closely related to the location and number of piezoelectric driving units.

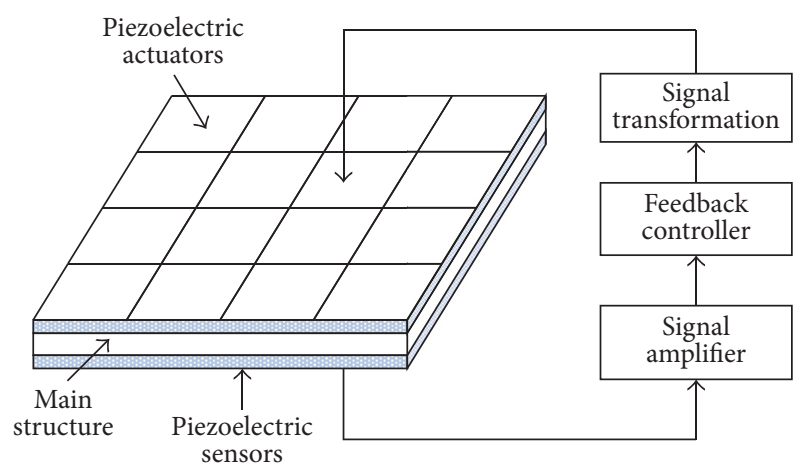

FIGURE 1: Active static shape control system of a piezoelectric intelligent plate.

Therefore, by changing the location, number, and input voltage of piezoelectric driving units, the overall displacement $\mathbf{q}$ in (11) may be changed to achieve shape control. A basic piezoelectric intelligent structure consists of a main structural layer, an upper layer, and a lower layer. If 1 piezoelectric layer is used as the sensing unit, the other layer is used as the driving unit, the output potential of the sensing unit is enlarged using the feedback control system, and the output voltage required by the driving unit is calculated via the feedback gain and control law. Then, active static shape control of the piezoelectric intelligent structure is fulfilled. The static shape system of a simple piezoelectric intelligent structure is shown in Figure 1.

\section{Specific Configuration of the Active Shape Controlled Genetic Algorithm}

The location and working voltage of piezoelectric actuators and sensors are the optimization goal of static shape control in a piezoelectric intelligent structure. Based on the finite element model of the overall structure, the finite elements shown in Figure 1 are discretized. Assume the piezoelectric actuators/sensors in the upper and lower surfaces of the finite elements in a unit are independently controllable, with the minimum variance of the actual and expected displacement of the structure being the target function, and with the location, number, and driving voltage of the piezoelectric plate being the variables of optimal design. Then, a computational program for the genetic optimization algorithm was compiled to seek the optimal solution that satisfies the expected shape of the piezoelectric intelligent structure.

Among the design variables, the location and number of piezoelectric plates are binary variables, while the driving voltage is a continuous variable. In this paper, a parameter cascade method was applied to code the location and driving voltage of the piezoelectric plate, and further compile codes to form the individual coding that represents the entire parameters.

Assume variables $\mathbf{X}_{1}$ and $\mathbf{X}_{2}$ represent the location and driving voltage of the piezoelectric plate, respectively. The 


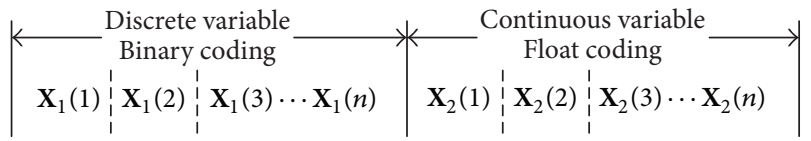

FIGURE 2: Individual bit string structure of parameter cascade coding.

binary-encoded method and the float-encoded method were applied to encode $\mathbf{X}_{1}$ and $\mathbf{X}_{2}$, respectively, and then

$$
\begin{aligned}
& \mathbf{X}_{1}=\left\{\gamma_{11}, \gamma_{12}, \gamma_{13}, \ldots, \gamma_{1 n}\right\}, \\
& \mathbf{X}_{2}=\left\{\gamma_{21}, \gamma_{22}, \gamma_{23}, \ldots, \gamma_{2 n}\right\},
\end{aligned}
$$

where $\mathbf{X}_{1}$ is a discrete variable. Using the binary-encoded method, $\gamma_{11}=0$ represents a situation where the unit finite element is not equipped with actuators/sensors, and $\gamma_{11}=1$ represents a situation where the unit finite element is equipped with actuators/sensors. In addition, $n$ represents the number of finite elements in the piezoelectric intelligent structure plate. Assuming the intelligent structure is equipped with $l$ pairs of actuators/sensors, then

$$
\sum_{i=1}^{n} \gamma_{1 i}=l
$$

Here, $\mathbf{X}_{2}$ is a continuous variable, float-encoded variable that directly reflects the actual value of the design variable and has the same length as variable $\mathbf{X}_{1}$. In addition, $\gamma_{2 i}$ is any real number within the given range of driving voltage: $[-a, a]$. Figure 2 illustrates the individual string structure that applies the parameter cascade code.

The genetic strategy was determined according to the form of the individual parameter cascade code, and the optimization model of piezoelectric intelligent structure shape control was established. The optimization algorithm system expression is as follows:

$$
\begin{array}{ll}
\min & G\left(\mathbf{X}_{1}, \mathbf{X}_{2}\right) \\
\text { S.t. } & R\left(\mathbf{X}_{1}, \mathbf{X}_{2}\right) \leq Q \\
\text { Fit. } & F(G),
\end{array}
$$

where $G\left(\mathbf{X}_{1}, \mathbf{X}_{2}\right)$ is the target function of the genetic algorithm optimization, $R\left(\mathbf{X}_{1}, \mathbf{X}_{2}\right)$ is the optimized restriction function, and $F(G)$ is the individual fitness function.

In real optimization of static shape control with different optimization objects, the corresponding optimization configuration differs. Taking into consideration the economy of controlling the cost, number, and voltage of actuators/sensors requires optimization. A minimum number of piezoelectric actuators/sensors and minimum driving voltage were used to obtain an ideal control shape. The genetic algorithm configuration is then described using a bi-objective optimization model:

$$
\begin{aligned}
& \min G_{1}\left(\mathbf{X}_{1}, \mathbf{X}_{2}\right)=\sum_{i=1}^{n} \mathbf{X}_{1}(i) \\
& \min G_{2}\left(\mathbf{X}_{1}, \mathbf{X}_{2}\right)=\sum_{i=1}^{n}\left|\mathbf{X}_{2}(i)\right|
\end{aligned}
$$

$$
\begin{array}{ll}
\text { S.t. } & R\left(\mathbf{X}_{1}, \mathbf{X}_{2}\right)=\sum_{j=1}^{m}\left(v_{O j}-v_{G j}\right)^{2} \leq R_{p} \\
\text { Fit. } & F_{1}\left(G_{1}\right)=n-G_{1}\left(\mathbf{X}_{1}, \mathbf{X}_{2}\right) \\
\text { Fit. } & F_{2}\left(G_{2}\right)=n a-G_{2}\left(\mathbf{X}_{1}, \mathbf{X}_{2}\right)
\end{array}
$$

where $n$ is the number of structure units; $m$ is the number of structure nodes; $v_{\mathrm{O} j}$ is the optimal displacement of each node; $v_{G j}$ is the targeted displacement for each node; and $[-a, a]$ is the space defined by the driving voltage. Furthermore, the target function of the genetic optimization algorithm $G_{1}\left(\mathbf{X}_{1}, \mathbf{X}_{2}\right)$ represents the configuration number of piezoelectric plates, $G_{2}\left(\mathbf{X}_{1}, \mathbf{X}_{2}\right)$ is the sum of absolute driving voltages of each plate, the restriction function $R\left(\mathbf{X}_{1}, \mathbf{X}_{2}\right)$ provides variance between the optimal and targeted shapes that satisfies control precision $R_{p}$, and the fitness functions $F_{1}\left(G_{1}\right)$ and $F_{2}\left(G_{2}\right)$ are the differences between target functions $G_{1}$, and $G_{2}$ and their theoretical maximum values, respectively.

Another optimization configuration method uses a given number of piezoelectric actuators/sensors, and optimizing the configuration location and driving voltage of the piezoelectric plate to achieve the optimal shape control effect within the precision requirement range. The specific configuration method of the genetic algorithm is as follows:

$$
\begin{array}{ll}
\min & G_{1}\left(\mathbf{X}_{1}, \mathbf{X}_{2}\right)=\sum_{j=1}^{m}\left(v_{O j}-v_{G j}\right)^{2} \leq R_{p} \\
\min & G_{2}\left(\mathbf{X}_{1}, \mathbf{X}_{2}\right)=\sum_{i=1}^{n}\left|\mathbf{X}_{2}(i)\right| \\
\text { S.t. } & R\left(\mathbf{X}_{1}, \mathbf{X}_{2}\right)=\sum_{i=1}^{n} \mathbf{X}_{1}(i)=l \\
\text { Fit. } & F_{1}\left(G_{1}\right)=\frac{1}{1+G_{1}\left(\mathbf{X}_{1}, \mathbf{X}_{2}\right)} \\
\text { Fit. } & F_{2}\left(G_{2}\right)=n a-G_{2}\left(\mathbf{X}_{1}, \mathbf{X}_{2}\right),
\end{array}
$$

where $l$ is the given number of piezoelectric actuators/sensors, while the other variables are the same as those in (19). Under this configuration, the target function of the genetic algorithm $G_{1}\left(\mathbf{X}_{1}, \mathbf{X}_{2}\right)$ becomes the variance of the optimal shape and target shape, the restriction function $R\left(\mathbf{X}_{1}, \mathbf{X}_{2}\right)$ becomes the number of piezoelectric plates equal to the given value, and the fitness function $F_{1}\left(G_{1}\right)$ becomes the reciprocal of $1+G_{1}\left(\mathbf{X}_{1}, \mathbf{X}_{2}\right)$.

Referring to the specific operational procedure of the genetic algorithm and based on the finite element model of piezoelectric intelligent structure, a computational procedure was compiled using Matlab, and a series of genetic algorithm optimization procedures were designed to realize active control optimization of the piezoelectric intelligent structure.

\section{Numerical Analysis}

Given a static shape control of a cantilever plate with the upper and lower surfaces painted with piezoelectric 
TABLE 1: Property parameters of cantilever intelligent structural plate.

Main structure: $\mathrm{Al}$

Elastic module: $E=71.0 \mathrm{GPa}$; the Poison parameter: $v=0.3$; density: $\rho=2690 \mathrm{~kg} / \mathrm{m} 3$

Intelligent material: PZT-5H

Elastic parameter: $E=126.0 \mathrm{GPa}$; Poison parameter: $v=0.35$; density: $\rho=7500 \mathrm{~kg} / \mathrm{m}^{3}$

Elastic rigid parameter: $c_{11}=12.6 \times 10^{10} \mathrm{~N} / \mathrm{m}^{2}, c_{12}=7.95 \times 10^{10} \mathrm{~N} / \mathrm{m}^{2}, c_{13}=8.41 \times 10^{10} \mathrm{~N} / \mathrm{m}^{2}, c_{33}=11.7 \times 10^{10} \mathrm{~N} / \mathrm{m}^{2}, c_{44}=2.33 \times 10^{10} \mathrm{~N} / \mathrm{m}^{2}$;

Piezoelectric stress constant: $e_{31}=-6.5 \mathrm{C} / \mathrm{m}^{2}, e_{32}=23.3 \mathrm{C} / \mathrm{m}^{2}, e_{33}=17 \mathrm{C} / \mathrm{m}^{2}$;

Dielectric constant: $\epsilon_{11}=1.503 \times 10^{-8} \mathrm{C} \cdot \mathrm{V} / \mathrm{m}, \epsilon_{33}=1.503 \times 10^{-8} \mathrm{C} \cdot \mathrm{V} / \mathrm{m}$

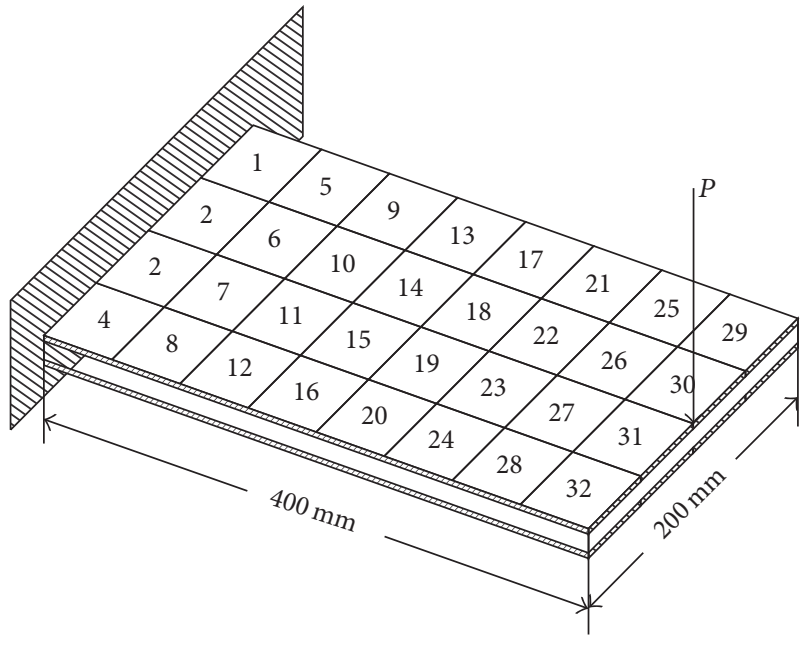

FIGURE 3: Finite element model of cantilever intelligent structural plate.

intelligent layers, the control theory of the finite shape and optimization control of the genetic algorithm were verified. The finite element model of the structure, which was divided into 32 finite rectangular plate elements, is shown in Figure 3. The upper and lower surfaces of the structure were symmetrically attached with piezoelectric thin film PZT-5H ( $1 \mathrm{~mm}$ thickness). The main structural material in the middle layer was Al, which had a thickness of $2.5 \mathrm{~mm}$. The property parameters of each material in the piezoelectric intelligent structure are listed in Table 1. Lastly, the middle point of the structural free end was affected by a constant load $P$, and the shape control target was defined as zero displacement of the free end. Structure optimization was then performed.

4.1. Configuration Analysis of the Genetic Algorithm: Optimizing the Number of Piezoelectric Actuators. According to (19), a minimum number of actuators should be used to satisfy structure shape control. Based on the finite element model and structure symmetry, 32 binary-encoded discrete design parameters G1 were set in the parameter cascade coding to represent configuration information regarding the location and number of piezoelectric actuators. The 32 float-encoded continuous design parameters G2 represented the working voltage of piezoelectric actuators. Working voltages were chosen within the range of $[-100,100]$, and the shape control precision (variance of actual displacement and expected displacement at the free end) was set as the restriction

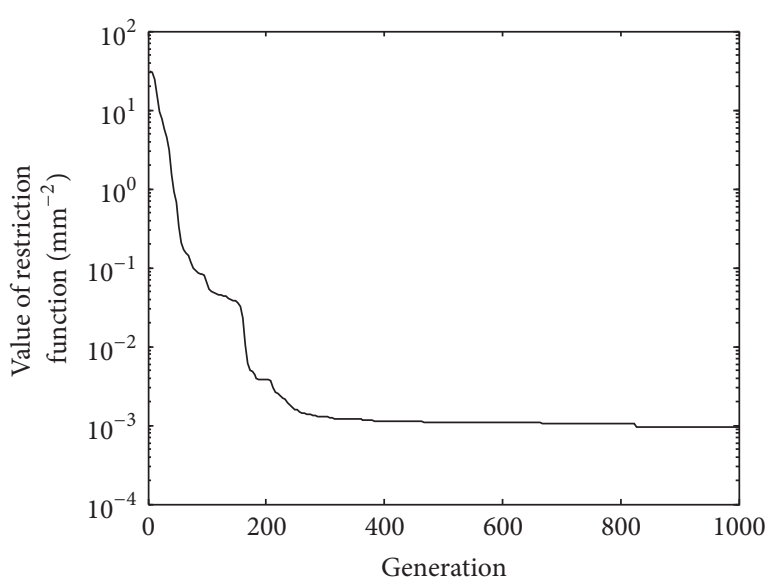

FIGURE 4: Variation of restriction function value.

function. The shape control precision requirement was set as less than $1 \times 10^{-3} \mathrm{~mm}^{2}$, the maximum number of generation as 1000 , the crossover possibility of the genetic algorithm as $P_{c}=0.625$, and the mutation probability as $P_{m}=1 / 64$. Then, the optimization calculation of the genetic algorithm was performed.

Firstly, we give random 32 binary-encoded discrete design parameters as the initial value. The displacement of the free end can be calculated through (10), and the restriction function $R\left(\mathbf{X}_{1}, \mathbf{X}_{2}\right)$ provides variance of actual displacement and expected displacement at the free end. If the shape control precision requirement is more than $1 \times 10^{-3} \mathrm{~mm}^{2}$, mutation will be applied to change the design parameters G1 and G2 to the new generation according to the mutation probability. The new generation design parameters will be used to start the new iteration.

After 1000 iterations, the optimization configuration that satisfied the shape control precision requirement was obtained. Figure 4 shows the performance tracking process of the restriction function value. The restriction function value rapidly converged and approached the required precision value after 350 iterations. After 1000 iterations, precision reached $9 \times 10^{-4} \mathrm{~mm}^{2}$. Figures 5 and 6 show the iterative evolution of the target function value and the fitness function value, respectively. Similarly, in the early stage of genetic optimization, the target function value immediately converged, and after 645 iterations, the number of configuration design parameters evolved from 32 to 6 , less than one-fifth of the initial design, which greatly saved the control cost. A configuration information diagram of each piezoelectric actuator 
TABLE 2: Optimal working voltage of piezoelectric actuators under optimal configuration.

\begin{tabular}{lc}
\hline Unit number & Working voltage $(\mathrm{V})$ \\
\hline 6 & 59.4 \\
10 & 72.1 \\
11 & -24.8 \\
15 & 42.5 \\
17 & 61.3 \\
20 & -36.7 \\
\hline
\end{tabular}

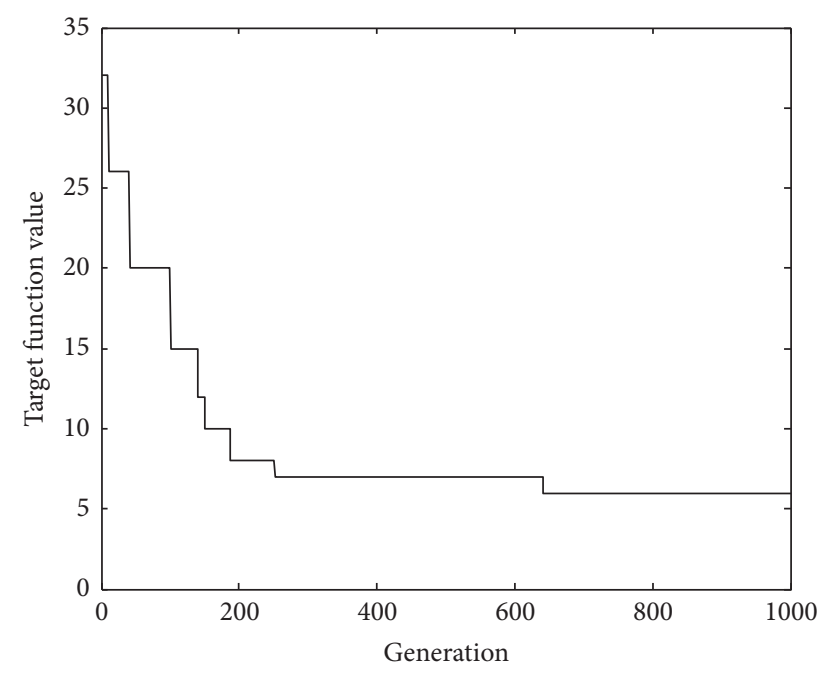

FIGURE 5: Variation of target function value.

in the iterative evolution process is shown in Figure 7, and Table 2 shows the working voltage of each piezoelectric actuator under the best configuration. It should be noted that variance of actual displacement and desired displacement at the free end was set as the restriction function, and then the symmetry of numerical results along the central axis of the beam could not be expected in genetic optimization. For example, the values of voltages between elements 10 and 11 , or 17 and 20 are derived to be positive or negative in Figure 7 and Table 2. Figures 8, 9, and 10 show shape diagrams of the piezoelectric intelligent cantilever at initial status, during evolution, and after optimization, respectively.

4.2. Configuration Analysis of Genetic Algorithm: Optimizing Shape Control Precision. With the number of actuators fixed, the optimum values of actuator location and working voltage were obtained via the optimization of the genetic algorithm to achieve the best control effect. The optimized genetic algorithm was performed according to (19), the parameter cascade encoding method was adopted, and 32 binary-encoded discrete design variables were chosen to represent configuration information regarding the location and number of piezoelectric actuators. The 32 float-encoded continuous design variables represented the working voltage within the range of $[-100,100]$. The shape control precision was selected as the target function (sum of squares of displacement and target displacement of 5 nodes in the free

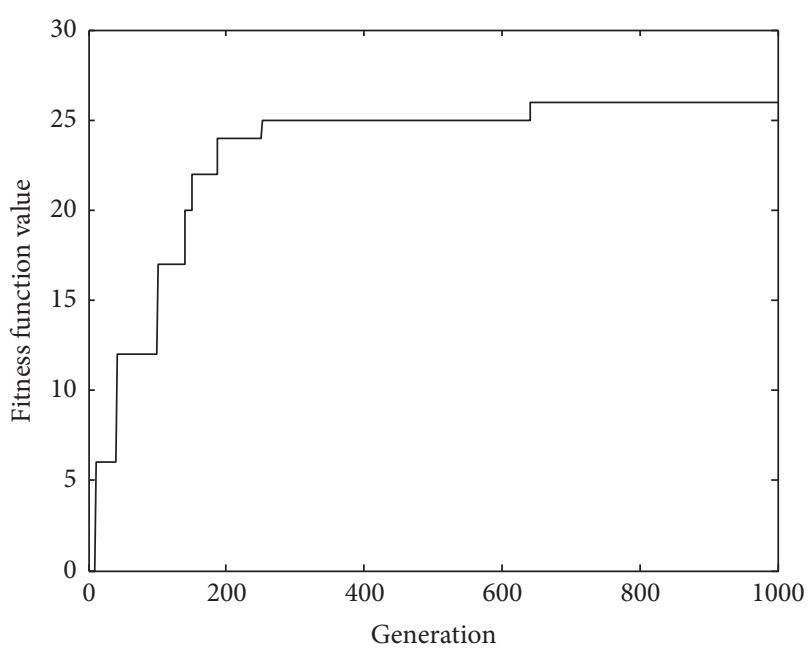

FIgURE 6: Variation of the fitness function value.

end after optimization), and the shape control precision requirement was set as less than $1 \times 10^{-5} \mathrm{~mm}^{2}$. Given 10 piezoelectric actuators, the restriction function value was 16 . Lastly, we assumed the maximum number of generations as 2000 , the crossover possibility of the genetic algorithm as $P_{c}=0.625$, and the mutation probability as $P_{m}=1 / 64$. Then, the optimization calculation of the genetic algorithm was performed.

Figure 11 shows the target function over 2000 iterations. The function rapidly converged during the initial iterative stages and approached the required precision after 400 iterations. Figure 12 shows the iterative variance of the corresponding fitness function. For a certain number of actuators, a relatively high control precision can be reached with proper optimization configuration. After 2000 iterations, the shape control precision of the piezoelectric actuators reached 5.21 $\times 10^{-6} \mathrm{~mm}^{2}$. Figure 13 shows the configuration diagram of the piezoelectric actuators at different evolution periods during iteration, while Figures 14 and 15 provide shape deformation diagrams of the piezoelectric intelligent plates during the optimization control process. Lastly, Table 3 shows the working voltage of each piezoelectric actuator under optimal configuration following iteration.

\section{Conclusions}

(1) Based on the dynamic finite element equation of piezoelectric intelligent structure, the static shape control principle of treating the piezoelectric material as an actuator was analyzed, demonstrating that the shape control effect is not only influenced by the parameters of the material itself, but also closely related to the location and number of piezoelectric driving elements. (2) By utilizing operation characteristics of the genetic algorithm (such as the embarrassingly parallel, random, and self-adaptive), each finite element of the piezoelectric intelligent structure was separated, and the computational and basic operational procedures of the optimal shape control genetic algorithm were designed to find the optimal solution that satisfied the expected shape. 


\begin{tabular}{|r|r|r|r|r|r|r|r|}
\hline 1 & 5 & 9 & 13 & 17 & 21 & 25 & 29 \\
\hline 2 & 6 & 10 & 14 & 18 & 22 & 25 & 30 \\
\hline 3 & 7 & 11 & 15 & 19 & 23 & 27 & 31 \\
\hline 4 & 8 & 12 & 16 & 20 & 24 & 28 & 32 \\
\hline
\end{tabular}

(a)

\begin{tabular}{|l|l|l|l|l|l|l|l|}
\hline 1 & 5 & 9 & 13 & 17 & 21 & 25 & 29 \\
\hline 2 & 6 & 10 & 14 & 18 & 22 & 25 & 30 \\
\hline 3 & 7 & 11 & 15 & 19 & 23 & 27 & 31 \\
\hline 4 & 8 & 12 & 16 & 20 & 24 & 28 & 32 \\
\hline
\end{tabular}

(c)

\begin{tabular}{|c|c|c|c|c|c|c|c|}
\hline 1 & 5 & 9 & 13 & 17 & 21 & 25 & 29 \\
\hline 2 & 6 & 10 & 14 & 18 & 22 & 25 & 30 \\
\hline 3 & 7 & 11 & 15 & 19 & 23 & 27 & 31 \\
\hline 4 & 8 & 12 & 16 & 20 & 24 & 28 & 32 \\
\hline
\end{tabular}

(b)

\begin{tabular}{|c|c|c|c|c|c|c|c|}
\hline 1 & 5 & 9 & 13 & 17 & 21 & 25 & 29 \\
\hline 2 & 6 & 10 & 14 & 18 & 22 & 25 & 30 \\
\hline 3 & 7 & 11 & 15 & 19 & 23 & 27 & 31 \\
\hline 4 & 8 & 12 & 16 & 20 & 24 & 28 & 32 \\
\hline
\end{tabular}

(d)

Figure 7: Configuration diagrams of piezoelectric actuators at different generations: (a) 40 generations; (b) 125 generations; (c) 200 generations; (d) 1000 generations.

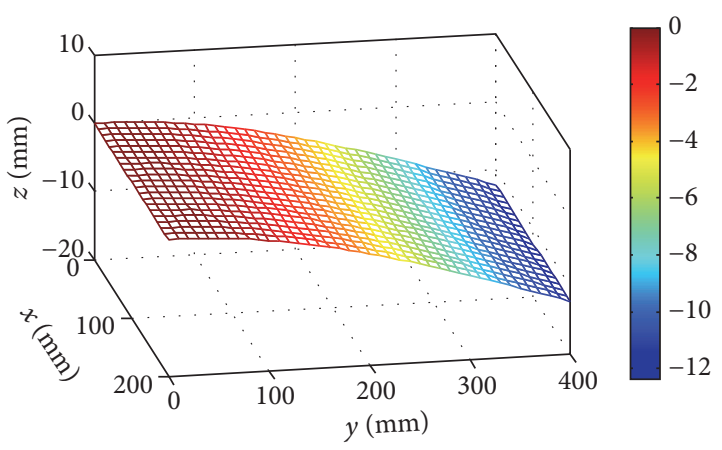

FIGURE 8: Initial shape without control.

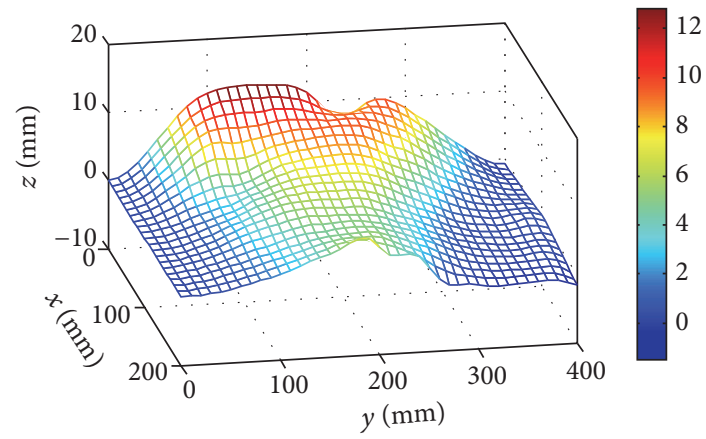

Figure 9: Controlled shape when evolution is at 125 generations.

(3) Based on the modified parameter cascade encoding method, we used the binary-encoded method and floatencoded method to code the location and driving voltage of the piezoelectric plates, respectively. We proposed a genetic algorithm optimization mode for shape control, and from the perspective of cost control economy and shape control precision, we then designed a computational program for the genetic algorithm. (4) Given a piezoelectric intelligent

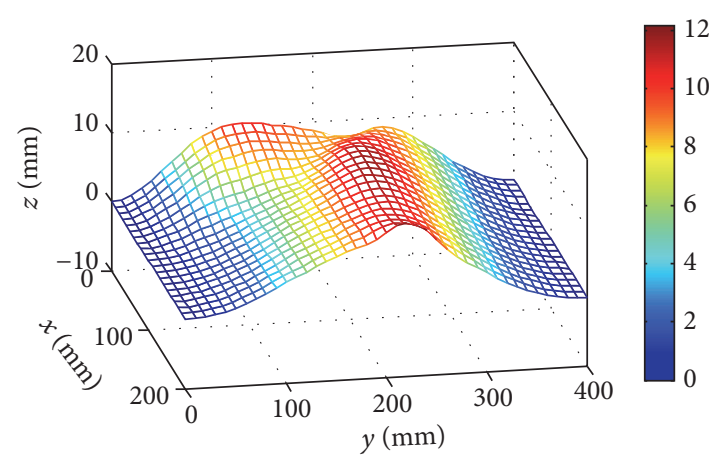

Figure 10: Controlled shape under optimal configuration.

TABLE 3: Optimal working voltage of piezoelectric actuator.

\begin{tabular}{lc}
\hline Unit number & Working voltage $(\mathrm{V})$ \\
\hline 6 & 67.2 \\
7 & 56.8 \\
9 & -19.2 \\
11 & 35.2 \\
14 & 41.2 \\
15 & 46.5 \\
16 & 21.6 \\
17 & -27.7 \\
18 & 41.5 \\
19 & 56.4 \\
22 & 61.4 \\
23 & 47.2 \\
\hline
\end{tabular}

cantilever with a load on 1 end, static shape optimization control results of piezoelectric materials based on the genetic algorithm were analyzed. Conclusively, our mathematical model and results indicated that active shape control with 


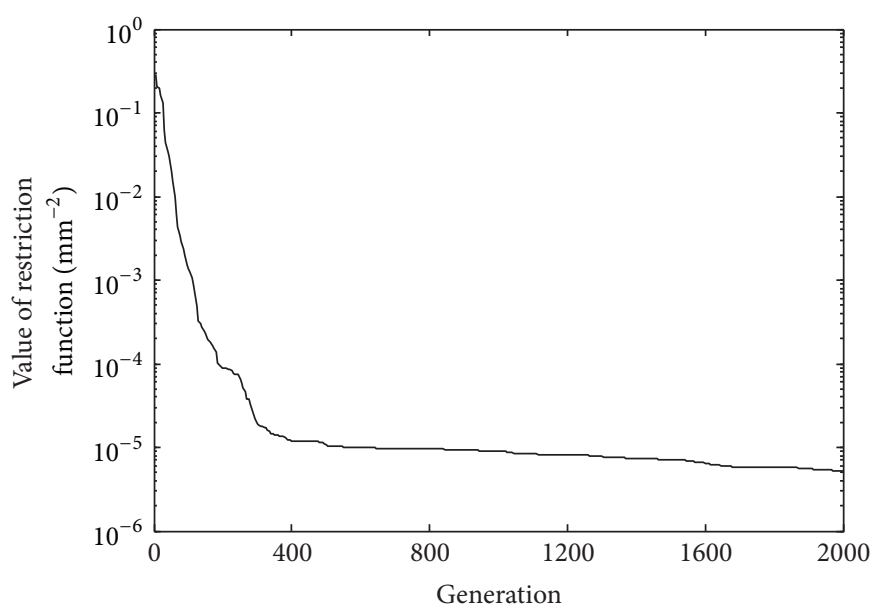

FIGURE 11: Variation of restriction function value.

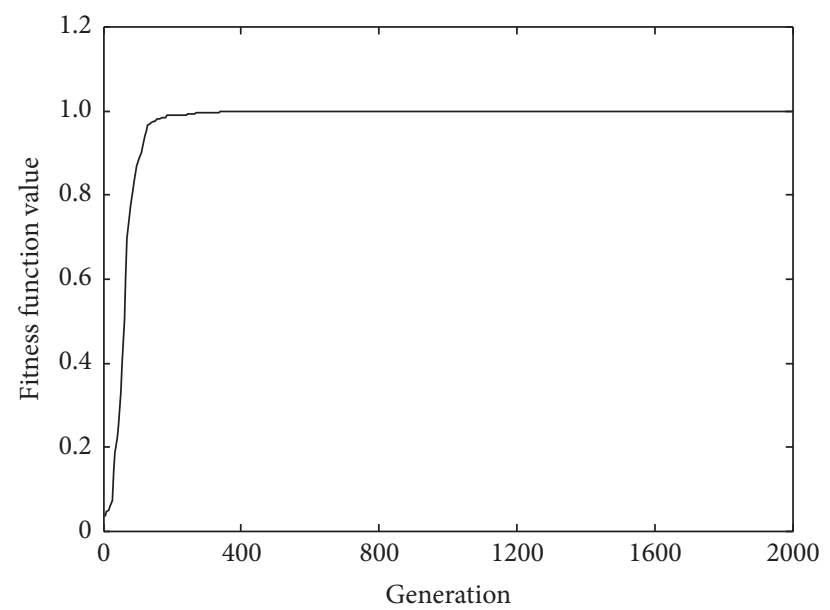

Figure 12: Variation of fitness function value.

\begin{tabular}{|c|c|c|c|c|c|c|c|}
\hline 4 & 8 & 12 & 16 & 20 & 24 & 28 & 32 \\
\hline 3 & 7 & 11 & 15 & 19 & 23 & 27 & 31 \\
\hline 2 & 6 & 10 & 14 & 18 & 22 & 25 & 30 \\
\hline 1 & 5 & 9 & 13 & 17 & 21 & 25 & 29 \\
\hline
\end{tabular}

(a)

\begin{tabular}{|r|r|r|r|r|r|r|r|}
\hline 4 & 8 & 12 & 16 & 20 & 24 & 28 & 32 \\
\hline 3 & 7 & 11 & 15 & 19 & 23 & 27 & 31 \\
\hline 2 & 6 & 10 & 14 & 18 & 22 & 25 & 30 \\
\hline 1 & 5 & 9 & 13 & 17 & 21 & 25 & 29 \\
\hline
\end{tabular}

(c)

\begin{tabular}{|c|c|c|c|c|c|c|c|}
\hline 4 & 8 & 12 & 16 & 20 & 24 & 28 & 32 \\
\hline 3 & 7 & 11 & 15 & 19 & 23 & 27 & 31 \\
\hline 2 & 6 & 10 & 14 & 18 & 22 & 25 & 30 \\
\hline 1 & 5 & 9 & 13 & 17 & 21 & 25 & 29 \\
\hline
\end{tabular}

(b)

\begin{tabular}{|r|r|r|r|r|r|r|r|}
\hline 4 & 8 & 12 & 16 & 20 & 24 & 28 & 32 \\
\hline 3 & 7 & 11 & 15 & 19 & 23 & 27 & 31 \\
\hline 2 & 6 & 10 & 14 & 18 & 22 & 25 & 30 \\
\hline 1 & 5 & 9 & 13 & 17 & 21 & 25 & 29 \\
\hline
\end{tabular}

(d)

FIGURE 13: Configuration diagrams of piezoelectric actuators at different evolution periods: (a) 20 generations; (b) 130 generations; (c) 320 generations; (d) 2000 generations. 


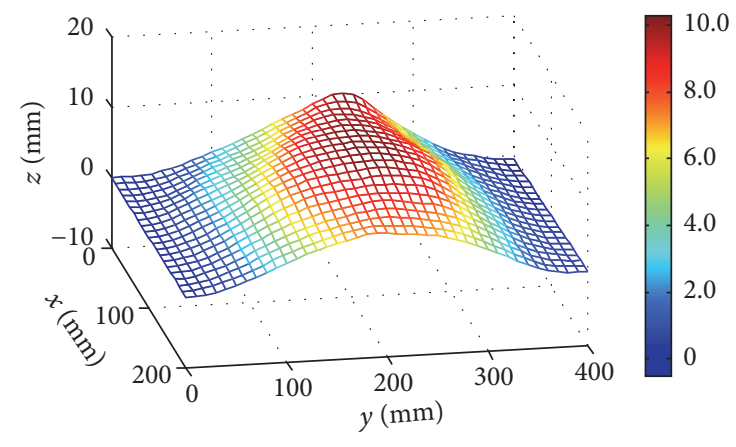

FIGURE 14: Controlled shape when evolution is at 320 generations.

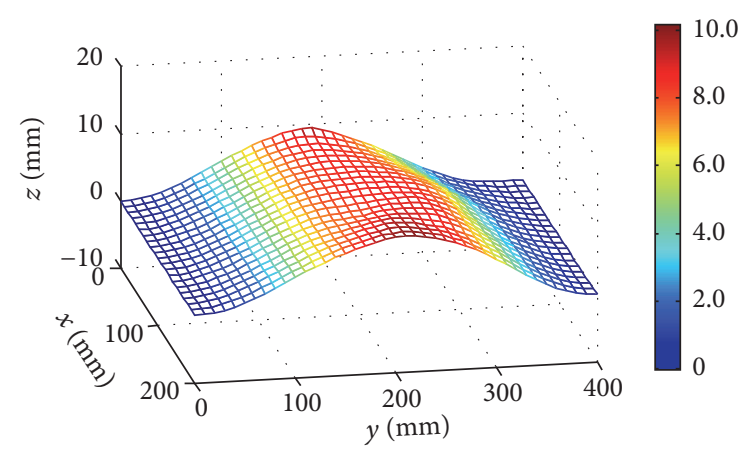

FIGURE 15: Controlled shape under optimal configuration.

high precision can be realized through optimization when using a certain number of piezoelectric actuators.

\section{Conflicts of Interest}

The authors declare that there are no conflicts of interest regarding the publication of this paper.

\section{Acknowledgments}

This work is partially supported by the National Natural Science Foundation of China (Grant no. 51505380), Shaanxi Science and Technology Innovation Project (Grant no. 2016KTZDGY06-01), and the "111 project" of China (Grant no. B13044).

\section{References}

[1] B. N. Agrawal, M. A. Elshafei, and G. Song, "Adaptive antenna shape control using piezoelectric actuators," Acta Astronautica, vol. 40, no. 11, pp. 821-826, 1997.

[2] H. Irschik, "A review on static and dynamic shape control of structures by piezoelectric actuation," Engineering Structures, vol. 24, no. 1, pp. 5-11, 2002.

[3] B. K. Wada, J. L. Fanson, and E. F. Crawley, "Adaptive Structures," Journal of Intelligent Material Systems and Structures, vol. 1, no. 2, pp. 157-174, 1990.

[4] P. Donthireddy and K. Chandrashekhara, "Modeling and shape control of composite beams with embedded piezoelectric actuators," Composite Structures, vol. 35, no. 2, pp. 237-244, 1996.
[5] S. Varadarajan, K. Chandrashekhara, and S. Aganval, "Adaptive shape control of laminated composite plates using piezoelectric materials," AIAA Journal, vol. 36, no. 9, pp. 1694-1698, 1998.

[6] C. Chee, L. Tong, and G. P. Steven, "Static shape control of composite plates using a curvature-displacement based algorithm," International Journal of Solids and Structures, vol. 38, no. 36-37, pp. 6381-6403, 2001.

[7] C. Chee, L. Tong, and G. P. Steven, "Piezoelectric actuator orientation optimization for static shape control of composite plates," Composite Structures, vol. 55, no. 2, pp. 169-184, 2002.

[8] J.-C. Lin and M. H. Nien, "Adaptive modeling and shape control of laminated plates using piezoelectric actuators," Journal of Materials Processing Technology, vol. 189, no. 1-3, pp. 231-236, 2007.

[9] D. Sun and L. Tong, "Static shape control of structures using nonlinear piezoelectric actuators with energy constraints," Smart Materials and Structures, vol. 13, no. 5, pp. 1059-1068, 2004.

[10] R. Barboni, A. Mannini, E. Fantini, and P. Gaudenzi, "Optimal placement of PZT actuators for the control of beam dynamics," Smart Materials and Structures, vol. 9, no. 1, pp. 110-120, 2000.

[11] J. C. Bruch Jr., J. M. Slossx, S. Adali, and I. S. Sadek, "Optimal piezo-actuator locations/lengths and applied voltage for shape control of beams," Smart Materials and Structures, vol. 9, no. 2, pp. 205-211, 2000.

[12] H. Zhang, B. Lennox, P. R. Goulding, and A. Y. T. Leung, "Float-encoded genetic algorithm technique for integrated optimization of piezoelectric actuator and sensor placement and feedback gains," Smart Materials and Structures, vol. 9, no. 4, pp. 552-557, 2000.

[13] S. Da Mota Silva, R. Ribeiro, J. Dias Rodrigues, M. A. P. Vaz, and J. M. Monteiro, "The application of genetic algorithms for shape control with piezoelectric patches - An experimental comparison," Smart Materials and Structures, vol. 13, no. 1, pp. 220-226, 2004.

[14] E. P. Hadjigeorgiou, G. E. Stavroulakis, and C. V. Massalas, "Shape control and damage identification of beams using piezoelectric actuation and genetic optimization," International Journal of Engineering Science, vol. 44, no. 7, pp. 409-421, 2006.

[15] IEEE Standard on Piezoelectricity, American National Standards Inst./IEEE Standard, 176-1987, New York, 1988.

[16] H. S. Tzou and C. I. Tseng, "Distributed piezoelectric sensor/actuator design for dynamic measurement/control of distributed parameter systems: A piezoelectric finite element approach," Journal of Sound and Vibration, vol. 138, no. 1, pp. 17-34, 1990. 

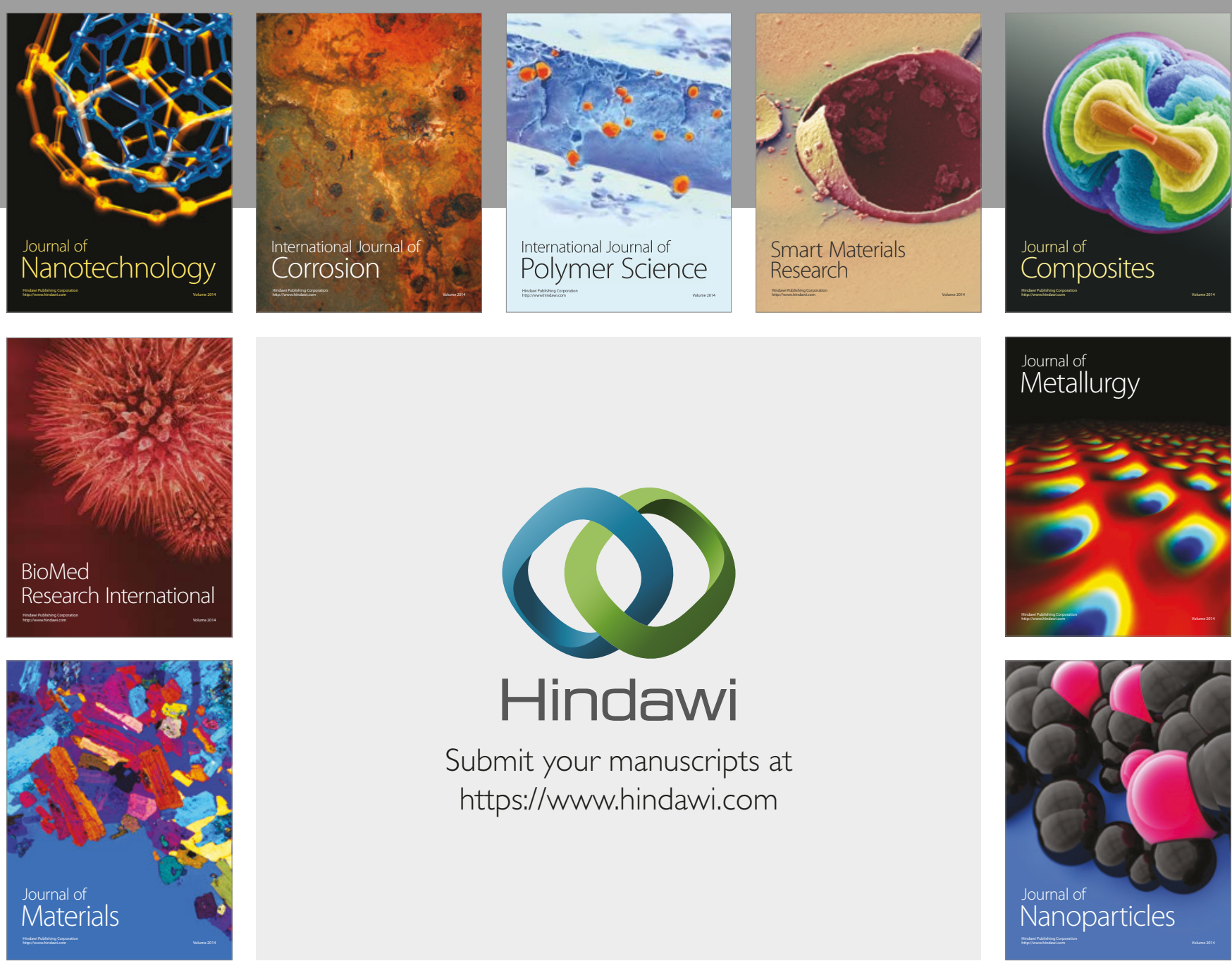

\section{Hindawi}

Submit your manuscripts at

https://www.hindawi.com
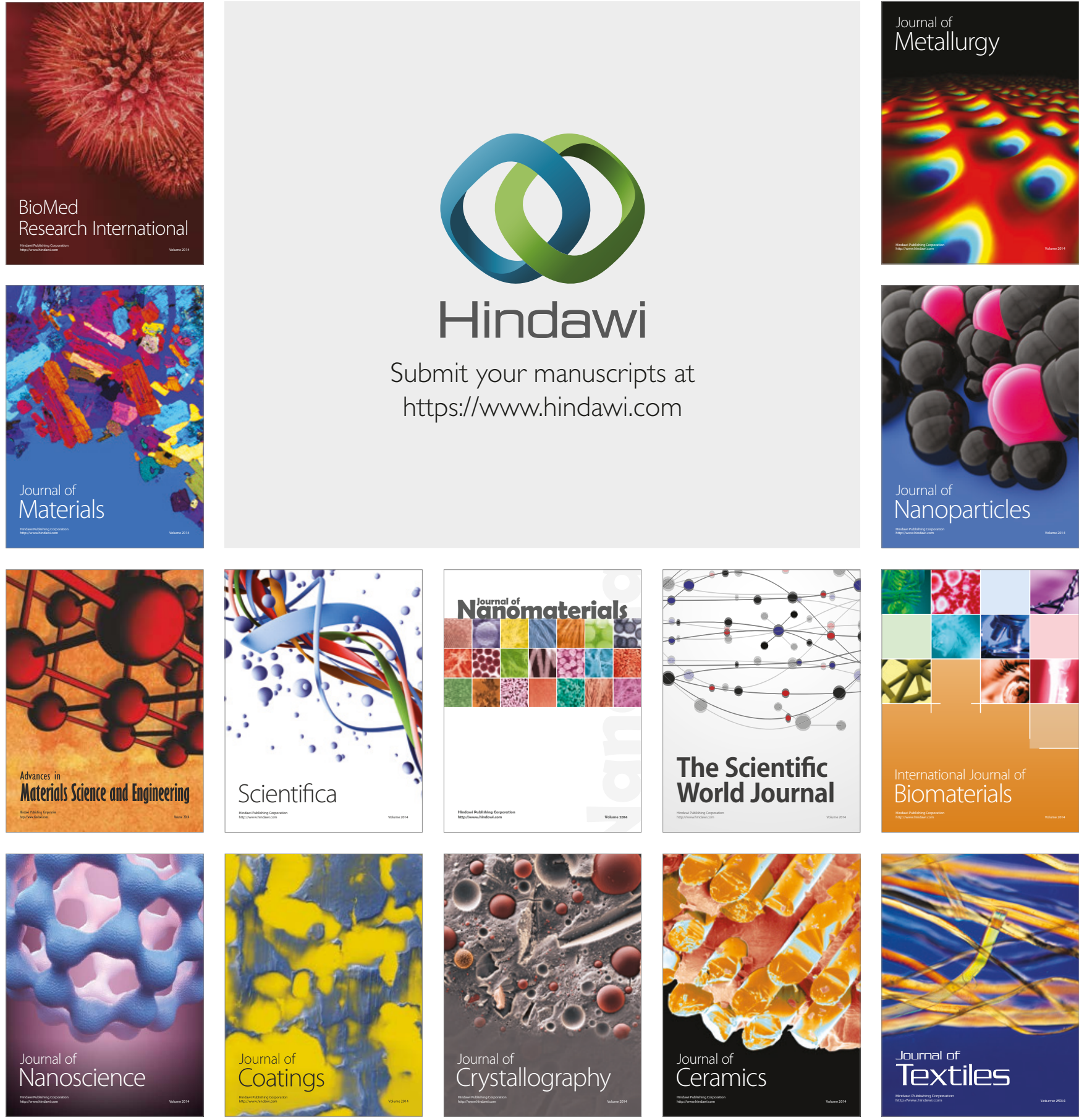

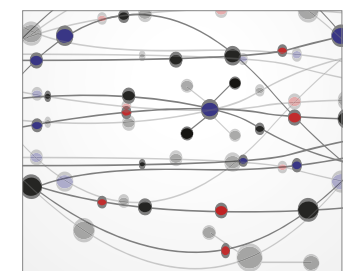

The Scientific World Journal
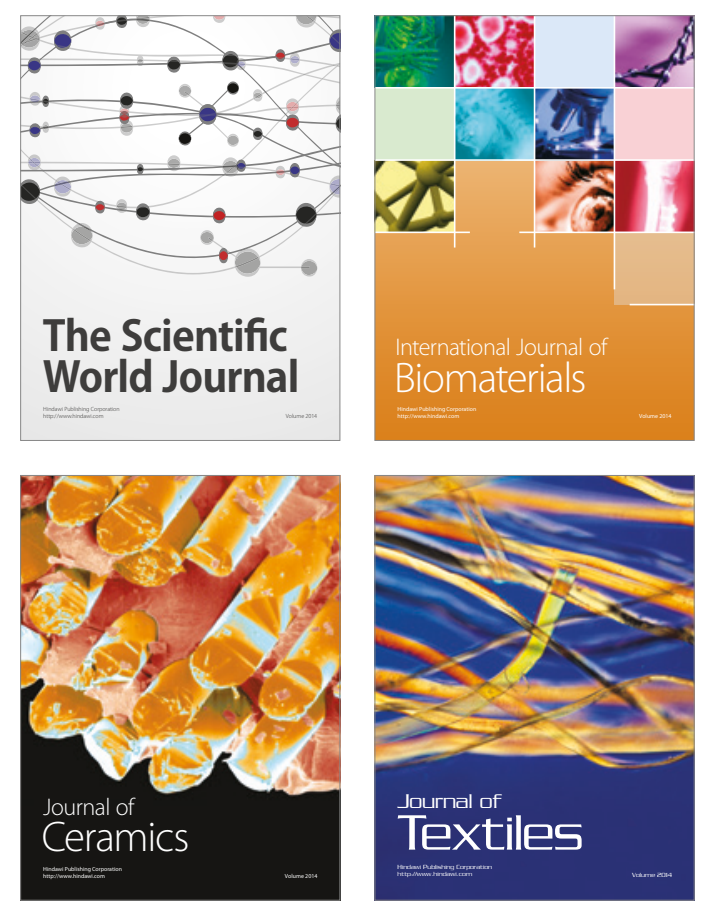